\title{
A randomised controlled pilot and feasibility study of music therapy for improving the quality of life of hospice inpatients
}

Sam Porter ${ }^{1}$, Tracey McConnell², Lisa Graham-Wisener ${ }^{3}$, Joan Regan³ ${ }^{3}$ Miriam McKeown³ ${ }^{3}$ Jenny Kirkwood ${ }^{4}$, Mike Clarke ${ }^{5}$, Evie Gardner ${ }^{6}$, Saskie Dorman ${ }^{7}$, Kerry McGrillen $^{3}$ and Joanne Reid ${ }^{8^{*}}$

\begin{abstract}
Background: Evidence about the effectiveness of music therapy for improving the quality of life of palliative care patients is positive but weak in terms of risk of bias.

Methods: This study aimed to determine the feasibility of a randomised controlled trial to evaluate the effectiveness of music therapy for improving the quality of life of hospice inpatients, as measured by the McGill Quality of Life questionnaire. Objectives included recruitment of 52 participants over 12 months and provision of data to support the calculation of the required sample size for a definitive randomised trial, taking into account the retention rates of recruited participants; and evaluation of the viability of the intervention and the acceptability of the assessment tool. The design was a single-centre, researcher-blinded randomised pilot and feasibility study involving two parallel groups. Participants were recruited from one inpatient hospice unit in Northern Ireland. Eligibility criteria were an Eastern Cooperative Oncology Group performance status of two or lower and an Abbreviated Mental Test score of seven or more. Consenting patients were randomly allocated to the intervention or control group using a 1:1 allocation ratio. The intervention group received up to six individual music therapy sessions over 3 weeks in addition to usual care. The control group received usual care only.
\end{abstract}

Results: Fifty one participants were recruited over 12 months. Twenty five were allocated to the intervention group and 26 to the control group. Seventy one percent of participants were lost to follow up by week 3, the proposed primary endpoint. The primary endpoint was moved from week 3, when $71 \%$ were lost to follow up to week 1 , when 33\% were lost. The McGill Quality of Life questionnaire was generally acceptable to participants. In order to detect a small to moderate effect size of 0.3, a fully powered study would require the recruitment of 698 participants.

Conclusions: A Phase III randomised controlled trial to evaluate the effectiveness of music therapy in improving the quality of life of hospice inpatients is feasible.

Trial registration: ClinicalTrials.gov: NCT02791048. Registered 6 June 2016.

Keywords: Music therapy, Palliative, Hospice, End-of-life, Quality of life, Pilot, Feasibility

\footnotetext{
* Correspondence: j.reid@qub.ac.uk

${ }^{8}$ School of Nursing and Midwifery, Queen's University Belfast, Belfast,

Northern Ireland

Full list of author information is available at the end of the article
}

(c) The Author(s). 2018 Open Access This article is distributed under the terms of the Creative Commons Attribution 4.0 International License (http://creativecommons.org/licenses/by/4.0/), which permits unrestricted use, distribution, and reproduction in any medium, provided you give appropriate credit to the original author(s) and the source, provide a link to the Creative Commons license, and indicate if changes were made. The Creative Commons Public Domain Dedication waiver (http://creativecommons.org/publicdomain/zero/1.0/) applies to the data made available in this article, unless otherwise stated. 


\section{Background}

Music therapy is defined as the use of music and sounds as part of a developing relationship between the patient and therapist to support and improve physical, mental and spiritual well-being [1]. At its introduction to palliative care in the 1970s, music therapy involved 'receptive' approaches where the patient could passively listen to music, and 'recreative' approaches where the patient could sing or play previously composed music [2-4]. Since then, it has developed to include improvisational music [5] and song writing [6], both of which involve greater creative input from the patient.

The effectiveness of music therapy in ameliorating a wide range of psychological and physical problems associated with palliative care has been reported in the literature [7]. This includes lowering levels of stress and anxiety, and improving mood, relaxation, overall wellbeing and attitude to life, along with reducing pain levels [8-10]. However, while several studies have suggested that music therapy may improve the quality of life of palliative care patients, many of them had a high risk of bias [10]. As a consequence, evidence about the effectiveness of music therapy for improving the quality of life of palliative care patients remains equivocal and this is an area of important uncertainty for the care of these patients. It has been suggested that this lack of robust evidence can at least partially explain why, while the availability of music therapy for palliative and end-of-life care patients is increasing, funding for it tends to be inconsistent [11].

There are a number of practical considerations that explain the lack of robust randomised trials in this area. Firstly, there are inherent challenges, such as high levels of attrition as a result of the deaths of participants, to conducting randomised trials with palliative care populations [12, 13]. Secondly, challenges such as clinical gatekeepers' lack of engagement with what they may see as a marginal 'complementary' therapy present additional difficulties to those engaging in experimental approaches to music therapy research [14].

\section{Aims and objectives}

This study aimed to determine the feasibility of a randomised controlled trial evaluating the potential effectiveness of music therapy for improving the quality of life of palliative care patients, and to pilot trial procedures. The study objectives were to:

a. Provide data to support the calculation of the required sample size for a definitive randomised trial. b. Evaluate the viability of delivering a 3 week music therapy intervention, taking into account the retention rates of recruited participants over that period.

c. Evaluate the acceptability of administering the McGill Quality of Life (MQoL) questionnaire to this population [15].

d. Evaluate the potential effectiveness of music therapy for improving quality of life.

e. Evaluate the potential effectiveness of music therapy for improving inter-familial communication.

f. Identify factors that help or hinder the implementation and sustainability of music therapy within a hospice setting.

Only objectives a-d are reported on in this paper. Objectives e and $f$, which make up part of the critical realist approach that is being taken to this evaluation [16], are reported separately [17].

\section{Methods}

A more detailed explanation of the study's procedures can be found in our published protocol [18]. In the following, we include changes to the protocol in response to interim findings.

\section{Study design}

This was a single-centre, researcher-blinded randomised feasibility study involving two parallel groups. The intervention group received individual music therapy in addition to usual care. The control group received usual care only.

\section{Randomization and masking}

Consenting patients were randomly allocated to the intervention or control group using a 1:1 allocation ratio. An independent statistician conducted blocked randomization with randomly permuted block sizes, which was used to fill opaque randomization envelopes. These were stored in the clinical investigator's locked filing cabinet at the hospice and used in sequence, thereby ensuring allocation concealment up to the point of randomization. To maintain researcher blinding, the treatment allocation was forwarded directly to the music therapist by the clinical investigator.

\section{Setting}

Participants were recruited from an 18-bed specialist palliative care unit in a hospice in Northern Ireland.

\section{Participants}

Patients were screened upon admission to the inpatient hospice unit by the clinical investigator. If they were deemed to have sufficient physical and mental capacity 
to take part (Eastern Cooperative Oncology Group (ECOG) performance status [19] of two or lower; Abbreviated Mental Test (AMT) Score [20] of seven or more), the clinical investigator provided them with information about the trial, including the participant information sheet. If they consented, she referred them to the researcher for potential recruitment. If, following further discussion with the researcher, the patient consented to participate, they were asked to sign an informed consent form. The researcher then informed the clinical investigator. Once recruited, the clinical investigator allocated patients to groups by opening the opaque envelopes in sequence.

After the first month, we recognised that recruitment was lower than expected. Clinical advice indicated that patients having an ECOG performance status that was worse than the initial exclusion threshold would still be capable of participating in the study, and might benefit from doing so. We therefore amended the protocol to include patients with an ECOG performance status of three or lower.

\section{Intervention}

Patients were randomly assigned to two groups. The control group received usual care, as deemed appropriate by the multidisciplinary hospice team. The experimental group received music therapy in an individual setting, delivered by a trained and registered music therapist, in addition to usual care.

A person-centred and creative music therapy approach which employed a systematic therapeutic process that included assessment, therapy and evaluation was adopted, guided by each patient's needs, interests, preferences and energy levels, and adapted accordingly in the moment [21]. During sessions, an interactive music therapeutic approach was used, whereby the client would engage by singing, playing or listening to known music, or extemporaneously create a melody, rhythm, song or instrumental piece with the therapist's support. The specific approach used in sessions was agreed with each participant.

Patients could choose to have a carer or significant other present during the intervention. If patients and carers wished, carers could be actively involved in the music therapy sessions.

Music therapy was conducted for up to $45 \mathrm{~min}$ twice a week over 3 weeks. In addition to standard clinical documentation, the music therapist completed an intervention manual [22] at the end of each session, including details on who chose the music and what strategy was used from the available suite of music therapy interventions (such as music listening, active music-making, improvising, and legacy work).

\section{Quantitative research instrument}

We used the McGill Quality of Life Questionnaire (MQoL) [15], which has been reported to have the best clinimetric quality rating, content validity, construct validity and internal consistency in a review of quality of life questionnaires for use in palliative care [23]. The MQoL is a 16 item questionnaire which is divided into five sub-measures with a varying number of questions for each sub-measure. The sub-measures are physical symptoms (3 questions), physical well-being (1), psychologic well-being (4), existential well-being (6), and support (2), with each question being scored from zero to ten by the patient. The final score is calculated as one fifth of the mean of the mean scores from each sub-measure. The higher the score, the better the quality of life of the patient and it has been suggested that a difference of 1 to 2 points in the overall score is equivalent to the difference between an average and a good day, and between a bad and an average day [24].

We also collected self-reported baseline data on the patients, including age, gender, ethnicity, marital status and their use of complementary therapies.

\section{Analysis of outcomes}

a. The capacity to recruit 52 patients to the study in a recruitment period of 12 months and to retain $70 \%$ of these to completion was recorded. 52 participants is a third more than the minimum recommended for good practice in a feasibility study [25] and a 30\% attrition allowance is recommended for a palliative care population [26]. These figures, along with input from practitioners and carers on what would be a clinically meaningful difference, were then used to support the calculation of the required sample size for a definitive RCT.

b. The viability of delivering a 3 week music therapy intervention was determined by attrition figures along with reasons for attrition.

c. The acceptability of the MQoL questionnaire was evaluated at each data collection point by the researcher, who requested verbal feedback from all patients in relation to the acceptability of the questionnaire's content and level of burden to complete. This verbal feedback was recorded and thematically analysed for any patterns in relation to acceptability. We also monitored and analysed the number of completed questionnaires along with reasons for non-completion using descriptive statistics.

d. Our initial intention was to assess potential effectiveness by determining the change over time in the MQoL, which was to be measured at baseline, at 
3 weeks (i.e. after completion of music therapy) and 5 weeks (i.e. 2 weeks after completion of music therapy). However, it became apparent that many patients were not surviving long enough after recruitment to reach the 3 week time point and we therefore instigated a MQoL follow-up at 1 week, making the change from baseline to this time point the primary outcome for the effectiveness assessment in this feasibility study. In the protocol, we had envisaged using analysis of covariance to compare the change in quality of life outcomes between the two randomised groups over time. However, for simplicity and given the high attrition rates beyond the first week, we report here the unadjusted mean differences between the groups for the change from baseline to week 1 . Results are shown with their associated standard deviations (SD) or 95\% confidence intervals (CI). In the absence of a formal power calculation, we advise caution when interpreting our results.

\section{Reporting}

The reporting of this study follows the CONSORT extensions for abstracts and for pilot and feasibility trials.

\section{Monitoring adverse events}

A Serious adverse events (SAE) form was included in each patient's case report form. As per protocol, any SAE was to be reported to the Principle Investigator within $24 \mathrm{~h}$, and reviewed by the Trial Steering Committee (TSC) at regular intervals throughout the trial. No adverse events were reported during the trial.

\section{Ethics approval and consent to participate}

Ethical approval was provided from the Office of Research Ethics Committee Northern Ireland (ORECNI) (reference number 16/NI/0058), which also provided approval for alterations to the protocol made during the study. Patients and staff provided written informed consent prior to participation in the study.

\section{Results}

\section{Recruitment, retention and sample size calculation}

We recruited and randomised 51 patients between June 2016 and June 2017 (the predesignated time point for ending recruitment), which was $98 \%$ of the original target of 52 participants. $43.2 \%$ of 118 patients deemed eligible for the study were recruited. Reasons for non-recruitment included those who declined $(n=28)$, early discharge $(n=12)$, non-availability of music therapist due to sickness/annual leave $(n=24)$, and music therapist at full capacity $(n=2)$.

One patient did not complete the baseline MQoL. The first nine patients (music therapy: 4; control: 5 ) were recruited before we introduced the outcome measurement at week 1 . The attrition rate for the week 1 outcome in the other 42 patients was $33 \%$. The attrition rates were much higher for outcome measurement at week 3 (71\% of 51 patients) and week 5 (92\% of 51 patients), primarily due to the death of the patient or because they were too unwell to complete the MQoL.

\section{Loss to follow-up}

We compared the baseline MQoL data for patients who did and did not complete the MQoL at week 1, to explore any differences between patients who were retained and those who were lost. Visual inspection of the data showed no differences between the randomised groups in the MQoL as a whole or within any of its sub-measures. Reasons for loss to follow-up are shown in the Consort Flow Diagram (Fig. 1). Table 1 shows the baseline characteristics of the randomised patients.

On the basis of data derived from self-assessed physical wellbeing at baseline, there was little evidence that the lowering of the ECOG score threshold to render patients who were less well eligible for recruitment led to a notable increase in attrition rates. There was no significant correlation between initial rating of physical wellbeing and likelihood of completion.

\section{Sample size calculation}

The difference in improvement between the groups was non-significantly in favour of music therapy: 0.30 (95\% CI: -0.45 to $1.05, p=0.43)$. Based on a two sample t-test, a sample size of 470 (235 per group) will have $90 \%$ power at a two-tailed significance level of 0.05 to detect a mean difference of 0.3 (pooled standard deviation $=1$ ) between groups, representing a small to medium effect size. Taking account of an attrition rate of $33 \%$, a fully powered study would be required to recruit 351 participants per group (see Table 2).

\section{Viability of three-week intervention}

The study protocol included a plan to recruit from hospice inpatients and outpatients. However, organisational difficulties relating to facilitating outpatients to attend music therapy sessions led to a decision to recruit exclusively from inpatients. One of the consequences of this decision was that the average life expectancy of potential participants was considerably reduced. This was reflected in the afore-mentioned high attrition rates at three and 5 week's follow-up. This attrition was problematic in relation to therapeutic intent, given that so many patients did not receive the intended dosage of the music therapy, and in assessing the effects of the therapy beyond the first week. We therefore concluded that a three-week intervention was not viable and, as noted above, that the primary outcome should be measured after 1 


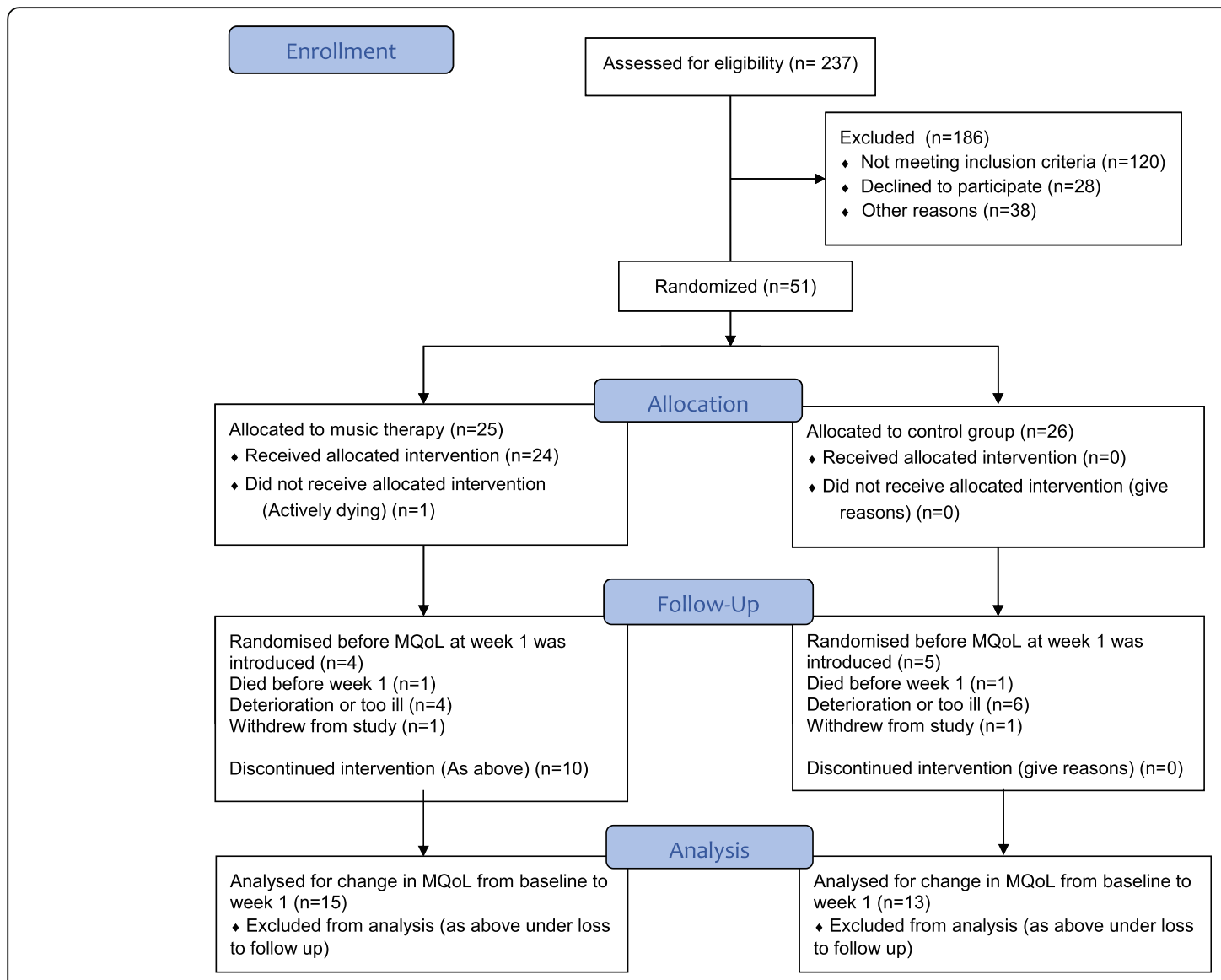

Fig. 1 Patient flow through the study

Table 1 Baseline characteristics of the randomised patients

\begin{tabular}{|c|c|c|c|c|c|c|}
\hline & Music therapy (a) & Control (a) & Total (a) & Music therapy (b) & Control (b) & Total (b) \\
\hline \multicolumn{7}{|l|}{ Gender } \\
\hline Female $[\mathrm{n}(\%)]$ & $20(80 \%)$ & $16(62 \%)$ & $36(71 \%)$ & $16(76 \%)$ & $13(62 \%)$ & $29(69 \%)$ \\
\hline Male $[n(\%)]$ & $5(20 \%)$ & $10(38 \%)$ & $15(29 \%)$ & $5(31 \%)$ & $8(38 \%)$ & $13(31 \%)$ \\
\hline Age (years) [mean (SD)] & $63(11.1)$ & $70.7(10.2)$ & $66.9(11.2)$ & $63.9(11.0)$ & $71.3(10.5)$ & $67.6(11.3)$ \\
\hline \multicolumn{7}{|l|}{ Ethnicity } \\
\hline White [n (\%)] & $25(100 \%)$ & $26(100 \%)$ & $51(100 \%)$ & $21(100 \%)$ & $21(100 \%)$ & $51(100 \%)$ \\
\hline \multicolumn{7}{|l|}{$\begin{array}{l}\text { Marital status (missing for one } \\
\text { control patient) }\end{array}$} \\
\hline Single $[\mathrm{n}(\%)]$ & $4(16 \%)$ & $3(12 \%)$ & $7(14 \%)$ & $3(\%)$ & $2(\%)$ & $5(\%)$ \\
\hline Married/with partner [n (\%)] & $15(60 \%)$ & $15(58 \%)$ & $30(59 \%)$ & $12(\%)$ & $14(\%)$ & $26(\%)$ \\
\hline Divorced [n (\%)] & $6(24 \%)$ & $2(8 \%)$ & $8(16 \%)$ & $6(\%)$ & $1(\%)$ & $7(\%)$ \\
\hline Widowed [n (\%)] & $0(0 \%)$ & $5(19 \%)$ & $5(10 \%)$ & $0(0 \%)$ & $3(\%)$ & $3(\%)$ \\
\hline $\begin{array}{l}\text { User of other complementary } \\
\text { therapies [n (\%)] }\end{array}$ & 17 (68\%) & $10(38 \%)$ & $27(53 \%)$ & $14(67 \%)$ & $7(33 \%)$ & $21(50 \%)$ \\
\hline Baseline MQoLMQoL [mean (SD)] & $6.1(1.2)$ & $6.0^{\mathrm{a}}(1.4)$ & $6.1^{a}(1.3)$ & $6.0(1.3)$ & $6.1^{\mathrm{a}}(1.4)$ & $6.0^{\mathrm{a}}(1.3)$ \\
\hline
\end{tabular}

(a) All 51 recruited patients; (b) 42 patients recruited after introduction of MQoLMQoL at week 1

a Excludes one patient who did not complete the baseline MQoLMQ 
Table 2 Change from baseline to week 1 in MQoL and sub-measures

\begin{tabular}{llll}
\hline Baseline MQoL, overall and sub-measures [mean, (SD)] & Music therapy $(n=15)$ & Control $(n=13)$ & Mean difference $(95 \%$ Cl) \\
\hline MQoL overall & $0.5(0.9)$ & $0.2(1.1)$ & $0.30(-0.45,1.05)$ \\
Physical symptoms & $1.5(2.6)$ & $0.4(3.5)$ & $1.10(-1.21,3.41)$ \\
Physical well-being & $-2.2(3.8)$ & $-0.2(3.4)$ & $-2.00(-4.67,0.67)$ \\
Psychologic well-being & $1.0(1.6)$ & $0.4(2.3)$ & $0.60(-0.89,2.09)$ \\
Existential well-being & $1.7(1.5)$ & $0.0(2.5)$ & $1.70(0.14,3.26)$ \\
Support & $0.4(1.9)$ & $0.3(3.7)$ & $0.10(-2.13,2.33)$ \\
\hline
\end{tabular}

week. This decision was supported by evidence that a one-week intervention had the potential to be therapeutically effective [27, 28]. Therefore, with approval from the Research Ethics Committee, we amended the protocol during the study to include an additional and earlier follow-up visit after 1 week (after one to two sessions of music therapy) to assess which time-frame is most feasible for an inpatient population at an advanced stage of their illness.

\section{Acceptability of MQoL questionnaire}

Feedback from participants on the acceptability of the MQoL questionnaire was encouraging. Only two participants questioned the pertinence of the information being requested. In terms of burden, only one participant declined to fill out the baseline questionnaire (6\% overall). Two patients withdrew from the study. The main issue of concern was the burden that follow-up administration of the questionnaire placed on participants, given the number of people identified who did not fill out questionnaires at follow-up because they were too ill.

\section{Potential effectiveness}

As expected in a feasibility study, the change from baseline to week 1 was not statistically significantly different between the music therapy and the control group. Among the patients who completed both the baseline MQoL and the MQoL at week 1, those in the intervention group $(n=15)$ showed a mean improvement of 0.5 points (SD: 0.9 ), while those in the control group $(n=13)$ showed a mean improvement of 0.2 points (SD: 1.1). The difference between the groups was non-significantly in favour of music therapy: 0.30 (95\% CI: -0.45 to $1.05, p=0.43)$. Table 2 shows the overall results and the results for the sub-measures in the MQoL. Although most of these are not significantly different between the randomised groups, there was a notable improvement in existential well-being for the music therapy group compared to the control group, and a notable disimprovement in significant disimprovement in physical well-being. However, as noted above these results needs to be interpreted with care and do not provide definitive evidence of a benefit or harm for music therapy in these aspects of patient well-being.

\section{Discussion}

\section{Recruitment, retention and sample size calculation}

While the target sample size was almost attained, the study identified a significant inhibitor to successful recruitment - the unavailability of a music therapist to carry out the intervention, which accounted for $39.4 \%$ of those deemed eligible for participation but not recruited. This indicates the need in any future trials for the logistical capacity of music therapy provision to be sufficiently robust to ensure that it is available to all participants who consent and who are randomly allocated to the intervention arm.

The study also identified major problems in relation to retention, with $71 \%$ of patients being lost to follow up at week 3 and 92\% at week 5; an attrition rate due overwhelmingly to their death or physical deterioration. Clearly, such retention rates indicate that an RCT based on these follow-up time points would not be feasible. In contrast, the attrition rate at week 1 was $33 \%$, marginally above the attrition rate pre-estimation of $30 \%$.

When considered in the context of recruitment rates in this study, the calculation that a fully-powered study would require the recruitment of 702 participants indicates the need for a multi-centre study that includes larger sites. The pilot study recruited 51 participants over 12 months in an 18 bed hospice. However, 26 non-recruitments were due to lack of music therapy resources, which in turn were due to limited funding for the study. A conservative assumption that $50 \%$ of those not recruited due to lack of music therapy resources could have been recruited if the adequate resources had been available gives a recruitment estimate of 64 participants per 18 beds per year. We therefore estimate that with access to sites with a total number of 100 beds, and with sufficient resources to ensure the consistent availability of music therapists, the sample size could be achieved over a recruitment period of 24 months. 


\section{Viability of intervention}

As noted above, the attrition rates recorded in this study indicate that a three-week intervention is not viable, but a one-week intervention is. However, while the participating music therapists concurred that a one-week intervention consisting of two music therapy sessions was a viable and potentially effective dosage, they reported that much of the first session offered was taken up with introducing the participants to music therapy, the music therapist, and to deciding what mode of music therapy would be most appropriate to their needs. This limited the time available for active therapy. This suggests that the most appropriate regimen would involve three sessions in a week, the first being primarily concerned with introductory activities. While the feasibility of this regimen was not tested in the pilot study, the palliative care clinicians and music therapists involved in the study concurred that, on balance, the extra burden to participants that an extra session involved (which they did not deem to be heavy) would be outweighed by the potential improvement in the effectiveness of the intervention.

\section{The acceptability of administering the MQoL questionnaire}

The main issue in relation to the acceptability of administering the questionnaire related to the fact that 12 out of the 14 patients lost to follow-up at week 1 withdrew because they were too ill to continue and therefore did not complete the follow-up questionnaire. However, given the clinical profile of the patient population involved in this study, this was expected (although the actual attrition rate was $10 \%$ higher than the pre-estimated rate). Given their physical deterioration, we do not believe that administering a shorter questionnaire would have had any significant impact on the number of patients completing. Given that the questionnaire was acceptable to the overwhelming majority of participants at baseline, we conclude that the MQoL is an acceptable instrument to use.

\section{Potential effectiveness}

While the study showed a non-statistically significant improvement of the overall scores from baseline to week 1 of the music therapy group compared to the control group, that outcome was the result of the aggregation of divergent scores for the individual domains, ranging from a significant improvement in existential well-being to a significant disimprovement in physical well-being. This disimprovement was a surprising result, not least because the closely related domain of physical symptoms showed a strong improvement. Moreover, a recent systematic review suggested that music therapy may be effective in helping to reduce pain in palliative care patients [10]. Nor have we been able to intuit any putative mechanisms by which music therapy might cause such a response. However, notwithstanding the need to treat this result with caution because of the size of the pilot study, it cannot be ignored. It is therefore important that any future, fully powered study should include both the overall quality of life result and the results of the individual domains as outcomes. Returning to the issue of the burden of the questionnaire, this conclusion underlines the importance of administering the complete questionnaire, rather than 'cherry-picking' those domains where improvement might be expected.

\section{Strengths and weaknesses}

The key strength of this study was its ability to demonstrate, in light of the well-documented difficulties of recruiting to music therapy research and the inherent problems of conducting research with a palliative care population, that a large-scale RCT to evaluate the effectiveness of music therapy in palliative care is feasible.

It also clearly demonstrated the weaknesses of the original protocol, most significantly in relation to recruitment and retention. In relation to recruitment, it demonstrated the importance of having sufficient human resources to ensure that everyone who consented to participate would be given the intervention if they were allocated to that group. The crucial role that resources play indicates the need for any future trial to incorporate a robust health economic analysis to ascertain the cost-effectiveness of music therapy compared to standard care.

In terms of retention, the large numbers lost to follow-up at week 3 and beyond demonstrated that a single week intervention, along with the administration of the MQoL at Day 7 after its baseline administration, was the only viable option. However, the use of a 7 day end point for data gathering, while enabling sufficiently powered analysis, would tell us nothing about whether or not music therapy was effective in improving the quality of life of surviving participants in the days and weeks after that point. It is therefore important that a future trial should incorporate evaluation of longer term effects. The difficulty with doing so is the already identified problem of attrition in this patient population, which rose to $71 \%$ at 3 weeks, making any statistically significant evaluation of effectiveness at this point unlikely. We therefore propose a compromise endpoint of 14 days at which time the MQoL should be re-administered to consenting patients. This should be supplemented by qualitative data to illuminate the experiences of participants and their close others.

\section{Conclusions}

This study has demonstrated the feasibility of conducting a Phase III RCT to evaluate the effectiveness of music therapy in improving the quality of life of 
palliative care patients in an inpatient hospice setting. We recommend that the next step for future research is to perform a fully powered trial using a revised protocol that takes into account the findings of this study. We recommend that the primary outcome of the trial should be the difference in change of MQoL from baseline to Day 7 between patient participants who received standard care with music therapy and those who received standard care alone. A secondary outcome should be the difference in change of MQoL between baseline and Day 14.

Robust findings from that trial would make a significant contribution to an evidence base that currently provides commissioners of palliative care services and therapies with equivocal information upon which to base their judgements about the inclusion or otherwise of music therapy in the palliative care armamentarium.

\section{Abbreviations}

AMT: Abbreviated Mental Test; ECOG: Eastern Cooperative Oncology Group; MQoL: McGill Quality of Life Questionnaire; RCT: Randomised controlled trial

\begin{abstract}
Acknowledgements
We wish to thank Dr. Simon Coulter (South Eastern Health and Social Care Trust), Dr. James Robertson (consultant music therapist), Corrina Grimes (Public Health Agency, DHSSPSNI) and Dori-Anne Finlay (Patient and Public Involvement representative) for providing guidance as independent members of the pilot trial steering committee. We would also like to acknowledge Dr. Emma Carduff (Marie Curie Hospice Glasgow), Daniel Thomas (CHROMA), Jo Godsal (CHROMA), Professor Peter Thomas (Bournemouth University), Dr. Sarah Thomas (Bournemouth University) and Mrs. Margaret Rose Norris (Patient and Public Involvement representative) of the main trial development committee for their advice. We acknowledge the crucial contribution made by Naomi Craig and Conall Dunlop who administered the music therapy intervention. Finally, we are very grateful to the patient and carer participants and staff at the Marie Curie Hospice Belfast for supporting the research.
\end{abstract}

\section{Availability of data or materials}

The datasets generated and analysed during the current study are available through contact with the corresponding author j.reid@qub.ac.uk.

\section{Funding}

This research was funded by a Northern Ireland Health and Social Care Research and Development Division Enabling Award. The funding body had no role in the design of the study, collection, analysis, interpretation of data or in writing the manuscript.

\section{Authors' contributions}

SP, TM, JoanneR, LG-W, JoanR, MM, KM and MC contributed to the trial design and protocol development. JK developed the intervention. TM, JoanneR, LG-W and KM were involved in data collection. MC, EG and SD statistically analysed the data. SP, TM, MC and JR drafted the manuscript and all authors contributed to and approved the final manuscript.

\section{Ethics approval and consent to participate}

Ethical approval was provided from the Office of Research Ethics Committee Northern Ireland (ORECNI) (reference number 16/NI/0058), which also provided approval for alterations to the protocol made during the study. Patients and staff provided written informed consent prior to participation in the study.

\section{Consent for publication}

Not applicable.

\section{Competing interests}

The authors declare that they have no competing interests.

\section{Publisher's Note}

Springer Nature remains neutral with regard to jurisdictional claims in published maps and institutional affiliations.

\section{Author details}

${ }^{1}$ Department of Social Sciences and Social Work, Bournemouth University, Bournemouth, England. ${ }^{2}$ School of Social Sciences, Education and Social Work, Queen's University Belfast, Belfast, Northern Ireland. ${ }^{3}$ School of Psychology, Queen's University, Belfast, Northern Ireland. ${ }^{4}$ Every Day Harmony Music Therapy, Belfast, Northern Ireland. ${ }^{5}$ School of Medicine, Dentistry and Biomedical Sciences, Queen's University Belfast, Belfast, Northern Ireland. ${ }^{6}$ Northern Ireland Clinical Trials Unit, Belfast, Northern Ireland. ${ }^{7}$ Poole Hospital NHS Foundation Trust, Poole, England. ${ }^{8}$ School of Nursing and Midwifery, Queen's University Belfast, Belfast, Northern Ireland.

Received: 16 March 2018 Accepted: 13 November 2018

Published online: 27 November 2018

\section{References}

1. Bunt L, Hoskyns S. The handbook of music therapy. Brunner- Routledge: East Sussex; 2002.

2. Munro S, Mount B. Music therapy in palliative care. Can Med Assoc J. 1978; 119:1029.

3. O'Kelly J, Koffman J. Multidisciplinary perspectives of music therapy in adult palliative care. Pall Med. 2007;21:235-41.

4. Clements-Cortes $\mathrm{A}$. The role of music therapy in facilitating relationship completion in end-of-life care/L'impact de la musicothérapie Sur le travail de séparation en fin de vie. Can J Music Ther. 2010;16(1):123-47.

5. Clements-Cortes A. The use of music in facilitating emotional expression in the terminally ill. Am J Hosp Palliat Med. 2004;21(4):255-60.

6. O'Callaghan C. The contribution of music therapy to palliative care. In: Hanks G, Cherny NI, Christakis NA, Fallon M, Kaasa S, Portenoy RK, editors. Oxford textbook of palliative medicine. 4th ed. Oxford: Oxford University Press; 1997. p. 214-21.

7. McConnell T, Porter S. Music therapy for palliative care: a realist review. Palliat Support Care. 2017;15(4):454-64.

8. Warth M, Kessler J, Hillecke TK, Bardenheuer HJ. Music therapy in palliative care-a randomized controlled trial to evaluate effects on relaxation. Dtsch Arztebl Int. 2015;112:788-94. https://doi.org/10.3238/arztebl.2015.0788.

9. Pothoulaki M, MacDonald R, Flowers P. An interpretative phenomenological analysis of an improvisational music therapy program for cancer patients. J Music Ther. 2012;49(1):45-67.

10. McConnell T, Scott D, Porter S. Music therapy for end-of-life care: an updated systematic review. Palliat Med. 2016;30(9):877-83.

11. Graham-Wisener L, Watts G, Kirkwood J, Harrison C, McEwan J, Porter S, Reid J, McConnell T. The provision and role of music therapists in palliative and end of life care in the UK: a service evaluation. BMJ Support Palliat Care. 2018; forthcoming.

12. Jordhøy MS, Kaasa S, Fayers P, Underland G, Ahlnr-Elmqvist M. Challenges in palliative care research; recruitment, attrition and compliance: experience from a randomized controlled trial. Pall Med. 1999;13(4):299-310.

13. Warth M, Kessler J, Koening J, Hillecke TKJ, Wormit AF, Bardenheuer HJ. Methodological challenges for music therapy controlled clinical trials in palliative care. Nord J Music Ther. 2015;24(4):344-71.

14. Porter S, McConnell T, Lynn F, McLaughlin K, Cardwell C, Holmes V. Recruiting participants for randomized controlled trials of music therapy: a practical illustration. J Music Ther. 2014;51(4):355-81.

15. Cohen SR, Mount BM, Strobel MG, et al. The McGill quality of life questionnaire: a measure of quality of life appropriate for people with advanced disease. A preliminary study of validity and acceptability. Pall Med. 1995;9:207-19.

16. Porter DS, McConnell T, Reid J. The possibility of critical realist randomised controlled trials. Trials. 2017a;18:133. https://doi.org/10.1186/s13063-017-1855-1.

17. Porter S, McConnell T, Clarke M, Kirkwood J, Hughes N, Graham-Wisener L, Regan J, McKeown M, McGrillen K, Reid J. A critical realist evaluation of a music therapy intervention in palliative care. BMC Palliat Care. 2017b:16. https://doi.org/10.1186/s12904-017-0253-5.

18. McConnell T, Graham-Wisener L, Regan J, McKeown M, Kirkwood J, Hughes N, Clarke M, Leitch J, McGrillen K, Porter S. Evaluation of the effectiveness of music therapy in improving the quality of life of 
palliative care patients: a randomised controlled pilot and feasibility study. Pilot Feasibil Stud. 2016;2:70.

19. ECOG-ACRIN Cancer Research Group. ECOG performance status. 2017. http://ecog-acrin.org/resources/ecog-performance-status. Accessed 04 Jan 2018.

20. Hodkinson HM. Evaluation of a mental test score for the assessment of mental impairment in the elderly. Age Aging. 1972;1:233-8.

21. Bradt J, Dileo C. Music therapy for end-of-life care. Cochrane Database Syst Rev. 2010;2:CD007169.

22. Robb SL, Burns DS, Carpenter JS. Reporting guidelines for music-based interventions. J Health Psychol. 2011;16:342-52.

23. Albers $\mathrm{G}$, Echteld $\mathrm{M}$, de Vet $\mathrm{H}$, et al. Evaluation of quality-of-life measures for use in palliative care: a systematic review. Pall Med. 2010. https://doi.org/10. 1177/0269216309346593.

24. Cohen SR, Mount BM. Living with cancer: "good" days and "bad" days - what produces them? Can the McGill quality of life questionnaire distinguish between them? Cancer. 2000;89(8):1854-65.

25. Lancaster GA, Dodd S, Williamson PR. Design and analysis of pilot studies: recommendations for good practice. J Eval Clin Pract. 2004;10:307-12.

26. Preston N, Fayers $\mathrm{P}$, Walters SJ, et al. Recommendations for managing missing data, attrition and response shift in palliative and end-of-life care research: part of the MORECare research method guidance on statistical issues. Pall Med. 2013. https://doi.org/10.1177/0269216313486952.

27. Hilliard RE. The effects of music therapy on the quality and length of life of people diagnosed with terminal cancer. J Music Ther. 2003:40:113-37 26.

28. Nguyen J. The effect of music therapy on end-of-life patients' quality of life, emotional state, and family satisfaction as measured by self-report. In: Master's thesis. USA: Florida State University; 2003.

Ready to submit your research? Choose BMC and benefit from:

- fast, convenient online submission

- thorough peer review by experienced researchers in your field

- rapid publication on acceptance

- support for research data, including large and complex data types

- gold Open Access which fosters wider collaboration and increased citations

- maximum visibility for your research: over $100 \mathrm{M}$ website views per year

At $\mathrm{BMC}$, research is always in progress.

Learn more biomedcentral.com/submissions 